\title{
PAPER Exploiting Visual Saliency and Bag-of-Words for Road Sign Recognition
}

\author{
Dan XU ${ }^{\dagger \dagger \dagger \mathrm{a})}$, Student Member, Wei XU ${ }^{\dagger \mathrm{b})}$, Zhenmin TANG ${ }^{\dagger c)}$, and Fan $\mathrm{LIU}^{\dagger \mathrm{d})}$, Nonmembers
}

\begin{abstract}
SUMMARY In this paper, we propose a novel method for road sign detection and recognition in complex scene real world images. Our algorithm consists of four basic steps. First, we employ a regional contrast based bottom-up visual saliency method to highlight the traffic sign regions, which usually have dominant color contrast against the background. Second, each type of traffic sign has special color distribution, which can be explored by top-down visual saliency to enhance the detection precision and to classify traffic signs into different categories. A bag-of-words (BoW) model and a color name descriptor are employed to compute the specialclass distribution. Third, the candidate road sign blobs are extracted from the final saliency map, which are generated by combining the bottom-up and the top-down saliency maps. Last, the color and shape cues are fused in the BoW model to express blobs, and a support vector machine is employed to recognize road signs. Experiments on real world images show a high success rate and a low false hit rate and demonstrate that the proposed framework is applicable to prohibition, warning and obligation signs. Additionally, our method can be applied to achromatic signs without extra processing.

key words: visual saliency, bag-of-words, road sign recognition, color names
\end{abstract}

\section{Introduction}

Road signs are used to regulate traffic, warn drivers, and provide useful information to help make driving safe and convenient. They play an important role in establishing wellordered traffic; therefore, traffic departments pay close attention to road sign inventory and management [1], [2]. One challenge for the task is that the road network and points of interest (POIs) of cities change frequently, which leads to the frequent modification of road signs [2]. Furthermore, many cities have no complete record about the setting of road signs. In this paper, we focus on road sign recognition for road sign inventory and management.

Vision-based traffic sign recognition methods can exploit beneficial characteristics of traffic signs, such as unique designs leading to small variations in shape and significant color contrast against the backgrounds [3]. Therefore,

\footnotetext{
Manuscript received December 19, 2013.

Manuscript revised April 11, 2014.

${ }^{\dagger}$ The authors are with the School of Computer Science and Engineering, Nanjing University of Science and Technology, Nanjing, 210094 China.

${ }^{\dagger \dagger}$ The author is with the School of Computer Science and Engineering, Jiangsu University of Science and Technology, Zhenjiang, 212003 China.

a) E-mail: xudan.zj@gmail.com

b) E-mail: njust.cst.xuwei@163.com

c) E-mail: tang.zm@mail.njust.edu.cn

d) E-mail: fanliu.njust@gmail.com

DOI: 10.1587/transinf.2013EDP7448
}

several color-based and shape-based sign detection models have been proposed to take advantage of these characteristics [4]-[6]. Although the vision-based models are convenient to implementation and have a good performance for recognizing road signs in certain conditions, there remain some challenges for successful recognition of a road sign in outdoor environments. First, the material of road signs may physically change over time and the color may degrade. Second, road signs will be influenced by the varying illumination condition when sampling. Moreover, there may be motion blur and abrupt contrast change caused by the motion of the camera.

Recently, some researchers have proposed saliency map models based on the biologically motivated selective attention mechanism, which imitate human-like early visual processing to overcome problems arising in complex environments [7], [8]. In this paper, we introduce the region based bottom-up saliency method to highlight the candidate road sign regions from the background. The image is first segmented into regions that are represented by color name descriptors [9]. The saliency value of a region depends on its color contrast with respect to other regions in the image. The bottom-up saliency method is not task dependent and just concerns the low-level features. However, top-down color attention is task driven and is dependent on the object categories. In our case, there are four categories of road signs, prohibition, warning, obligation and release of prohibition. Every category has special color information, as shown in Fig. 1. By learning the color information, category-specific color attention maps are constructed, which can be not only used to enhance detection precision but also to classify traffic signs into different categories.

Release prohibition signs consist of white and black colors; therefore, we denote them as achromatic signs. The detection of achromatic signs is another problem for researchers. HSI is a popular color space for road sign detection; however, the hue and saturation components do not contain enough information to segment white signs. Achromatic decomposition was used for white signs detection in [2], which needs additional computation. In this paper, we introduce the color name descriptor to represent an image, which displays a certain amount of photometric invariance and provides an added advantage of allowing the description of achromatic colors, such as white, gray and black [9].

There are many types of color and shape descriptors that have been used for road sign recognition. In this paper, we fuse color and shape cues, which are represented 


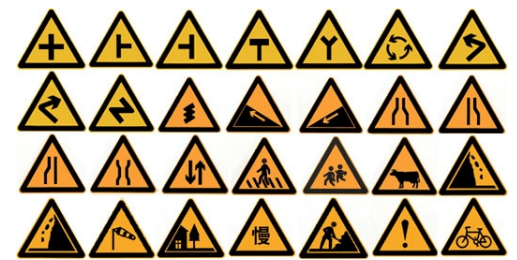

(a)

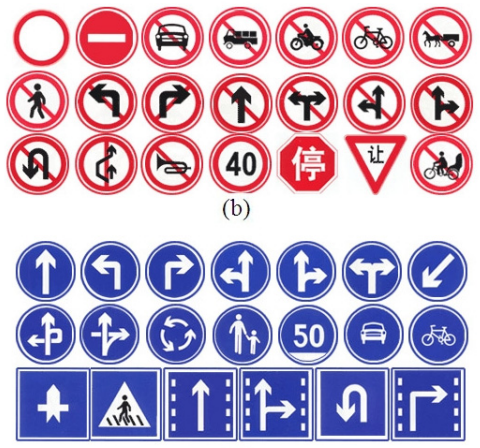

(c)

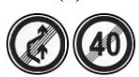

(d)

Fig. 1 Traffic sign data set.(a) Warning signs, (b) prohibition signs, (c) obligation signs and (d) achromatic signs.

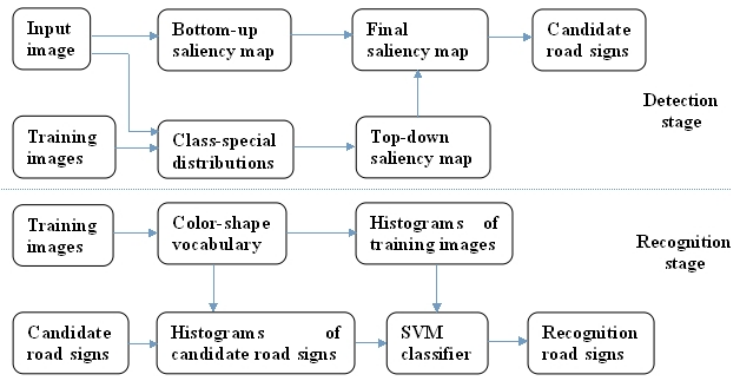

Fig. 2 Flowchart of our method.

by color name and SIFT descriptors, respectively, in the bag-of-words (BoW) model to form a color-shape vocabulary. Every road sign can be represented by a histogram that expresses the occurrence frequency of the visual words. Finally, the support vector machine (SVM) is employed to train a classifier to recognize each road sign. The overview of our method is shown in Fig. 2.

The paper is organized as follows. In Sect. 2, we discuss related work. Section 3 introduces the approach of road sign detection in detail. Recognition stage is discussed in Sect. 4. Experiments and analysis are given in Sect. 5. Section 6 finishes with concluding remarks.

\section{Related Work}

Road sign recognition has attracted wide attention and is becoming more important for driver assistant system and road sign management. To recognize road signs, various methods have been proposed in the last few decades that have shown promising results. The systems usually have been developed in two phases: the detection and recognition phases.

\subsection{Road Sign Detection}

The detection algorithms are normally based on shape or color segmentation, which can be classified into color-based and shape-based groups. Most existing road sign detection methods include color processing. Color thresholding segmentation is one of the simplest methods used for road sign detection, which classifies the pixels in an image into 'object pixels' and 'background pixels' in RGB space [5]. HIS transformation is employed in [2] to reduce the influence of illumination changes. The authors believed that the hue and saturation components of the HIS space are sufficient to isolate traffic signs in a scene working with fixed thresholds. Color Neural networks are trained to group and recognize patterns of colors, followed by extracting out the color road sign objects from the background [10].

Shape-based methods form the second main group of road sign detection techniques. Comparing to color-based approaches, shape-based techniques have to address imperfect shape problems and the sign appearance. Radial Symmetry Transform (RST) was first used for circle road signs detection and then extended to octagonal, square and triangular shapes [11], [12]. In [6], Barnes proposed a regular polygon detector using a posteriori probability approach based on locality and gradient information. Furthermore, some geometric features are used. The ratio between the square of the perimeter and the area (Peri2Area) and the number of sides (NumSides) are employed in [5] to classify the regions of interest (ROIs) into different regular polygons. Shape detection generally necessitates robust edge detection and/or matching algorithms to detect the relevant shapes.

The survey by Møgelmose et al. [13] provides detailed analysis of the most recent developments in road sign detection. Herein we discuss the road sign detection methods that are based on visual saliency. Recently, saliency detection has attracted more attention in computer vision because of its wide application [8], [14]. Itti et al. proposed a saliency model that computes saliency in each of several feature channels (e.g., color, intensity, orientation; saliency is then the relative difference between a region and its surrounding) in parallel and fuses them in a scalar map called the 'saliency map' [15]. Woong-Jae Won et al. proposed a road sign detection method based on the main idea of Itti's model. Because the road traffic signboards have dominant color contrast against backgrounds, they consider the color opponents and its edge information with center surround difference and normalization as a pre-processing before the saliency map is generated [14], [16]. Anh Cat Le Ngo et al. used traffic-sign colors to tune the bottom-up visual saliency from general object detection to traffic-sign detection [7].

\subsection{Road Sign Recognition}

Various features are available for road sign recognition in 
the vision literatures. The most popular feature is edges: edges can be directly obtained from the raw picture or from pre-segmented images. Edges are used as the only feature in [4], [17] and [18]. Although edges comprise the most popular feature choice, there are other options. Histogram of Oriented Gradient (HOG) is based on creating histograms of gradient orientations on patches of the image and comparing them to known histograms for the sought-after objects. Although it was first used to detect pedestrian in the image, it has been used for sign detection and classification in [19] and [20]. In addition, more esoteric features are used. Distance to bounding box (DtB) is used in [2] as a measure of distances from the contour of a sign candidate to its bounding box. Similarly, the FFT of shape signatures used in [21] is based on the distance from the shape center to its contour at different angles. Tangent functions, which are used in [22], calculate the angles of the tangents at various points around the contour.

Different techniques in image classification, such as Artificial Neural Networks (ANN), Support Vector Machines (SVM), Fuzzy measures, Genetic Algorithms (GA), Fuzzy Support Vector Machines (FSVM) and Genetic Algorithms with Neural Networks are being developed for road sign recognition and classification.

\section{The Proposed Detection Method}

Attention theories proposed by Itti suggest two stages of visual attention: fast, pre-attentive, bottom-up, data driven saliency extraction and slower, task dependent, top-down, goal driven saliency extraction [23]. We integrate bottomup, lower-level features with top-down, higher-level priors for road sign detection.

\subsection{Bottom-Up Saliency Detection}

Bottom-up saliency can be viewed from different perspectives. Contrast-based and uniqueness-based methods are two typical categories. We introduce a regional contrast saliency method where we first segment the input image into regions and then assign saliency values to them by computing their contrast with respect to other regions. Color name is first used for image segmentation and then employed as a color descriptor for region representation. The region contrast is measured by inter-regions distance among color name descriptors.

\subsubsection{Color Names}

Color names, or color attributes, are linguistic labels that humans assign to colors in their world. Berlin and Kay [24] in a linguistic study concluded that the English language contains eleven basic color terms: black, blue, brown, gray, green, orange, pink, purple, red, white and yellow. In computer vision, color attributes involve the assignment of linguistic color labels to pixels in an image. This requires a mapping between pixel values and color attributes. In [25],
Van de Weijer et al. learned the relationships between color names and the pixel values, $p(c \mid v)$, by training color names in real-world images ${ }^{\dagger}$.

$p(c \mid v)$ denotes the probabilities of color names for a given color value. Given a pixel $x, v(x)$ denotes its value in $L^{*} a^{*} b^{*}$ space, the probabilities of different color names to which it belongs can be represented as $\left(p\left(c n_{1} \mid v(x)\right), p\left(c n_{2} \mid v(x)\right), \cdots, p\left(c n_{11} \mid v(x)\right)\right)$ according to $p(c \mid v)$. For pixel assignment, $x$ is assigned to the color name that has max probability.

The color name descriptor $(\mathrm{CN})$ is defined as a vector containing every color name's probability for a given region $R$.

$$
C N=\left(p\left(c n_{1} \mid R\right), p\left(c n_{2} \mid R\right), \cdots, p\left(c n_{11} \mid R\right)\right)
$$

With

$$
p\left(c n_{i} \mid R\right)=\frac{1}{N} \sum_{x \in R} p\left(c n_{i} \mid v(x)\right)
$$

where $x$ stands for the pixel in region $R$, with total number of N. $v(x)$ is the pixel value in $L^{*} a^{*} b^{*}$ color space. $p\left(c n_{i} \mid v(x)\right)$ is the conditional probability of color name $i$ for a given $v(x)$ and can be obtained from the learned $p(c \mid v)$.

Color name descriptors display a certain amount of photometric invariance because several changes of a color are mapped into the same color name in the training stage. They also provide an added advantage of allowing the description of achromatic colors, such as black, gray and white, which are impossible to distinguish from a photometric invariance perspective [9]. Color names have been found to be a successful color feature for image classification [26].

\subsubsection{Regional Contrast Saliency Detection}

Color names can be used for pixel assignment, i.e., to assign a pixel to a special color. Color clusters are obtained after pixels assignment over the whole image, followed by segmenting each cluster into regions. Take the red cluster for example: the pixels belonging to red are set to 1 , all other pixels are set to 0 , and the cluster is segmented into regions in a binary image. The overview of the image segmentation is shown in Fig. 3. Note that assignment is computed based on individual pixels, which results in a large number of isolated points. Therefore, we employ median filtering after the pixel assignment, which reduces the number of regions significantly.

If a region is identified as salient, it at least has one feature different from the surroundings. We would like the feature descriptor to be discriminative, compact and invariant to illumination. Here, the color name descriptor is used to represent regions. Saliency of region $k$ in cluster $i$ is based on its contrast with respect to other regions $l$ in the image among color descriptors.

$$
S_{i k}=\sum_{r_{l} \neq r_{i k}} D_{c}\left(r_{l}, r_{i k}\right)
$$

\footnotetext{
${ }^{\dagger}$ http://lear.inrialpes.fr
} 


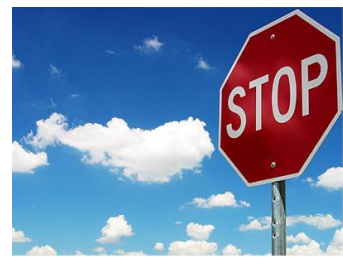

(a)

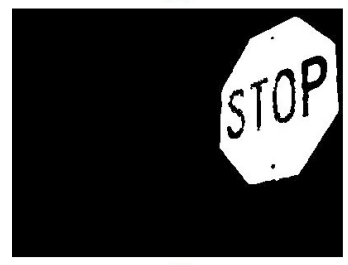

(c)

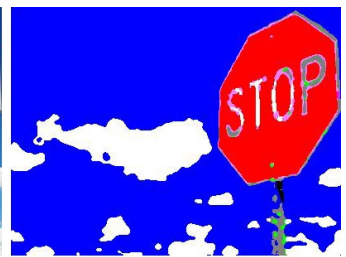

(b)

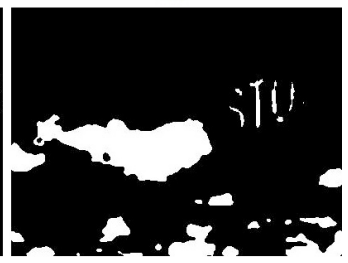

(d)
Fig. 3 Overview of image segmentation. (a) Original image, (b) color clusters after pixel assignment, (c) red regions and (d) white regions.

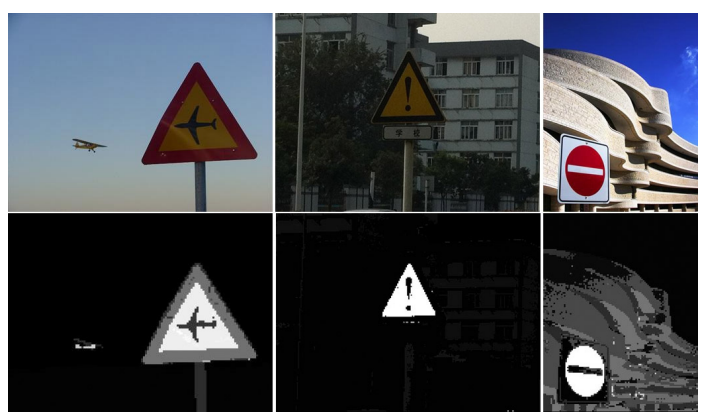

Fig. 4 Saliency maps produced by the bottom-up saliency detection method.

With

$$
D_{c}\left(r_{1}, r_{2}\right)=D\left(C N_{1}, C N_{2}\right)
$$

where $C N_{1}, C N_{2}$ are $\mathrm{CN}$ descriptors of $r_{1}, r_{2} . D\left(C N_{1}, C N_{2}\right)$ is the Euclidean distance between two color descriptors.

The saliency detection method based on regional contrast and color names is more effective for objects with vivid colors, such as flowers and road signs, or for objects with high contrast against the background color. Some saliency maps produced by the method are shown in Fig. 4.

\subsection{Top-Down Color Attention}

In contract to the common bottom-up saliency, top-down attention introduces higher-level features and prior knowledge into the objective function. In this study, road signs are divided into four categories and every category has special color information, as shown in Fig. 1. For each category, class-specific distribution is constructed by BoW model with training images to form the top-down saliency maps [27]. Then, the traffic signs are detected from the final saliency maps, which are generated by combining the bottom-up saliency maps with top-down saliency maps.

BoW has been used in computer vision in which an image can be represented by a sparse vector of occurrence

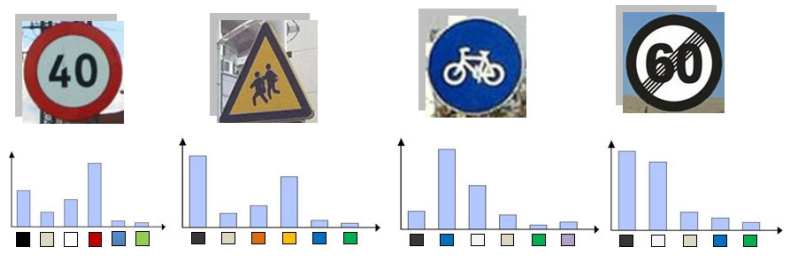

Fig. 5 Color histograms of different types of road signs.

counts of visual words in the vocabulary of local image features. Typically, BoW model consists of three stages, local feature extraction, vocabulary generation and image representation. First, for images $I^{i}(i=1, \cdots, N)$, a number of local features $f_{i j}\left(j=1, \cdots, M^{i}\right)$ are detected, where $M^{i}$ is the total number of local features in image $i$. Generally, the local features are represented in visual vocabularies, which describe various image cues, such as shape, texture and color. In the detection stage, we pay our attention on color cue and express local features by color name descriptors. Then, a universal visual vocabulary representing all road sign categories is computed by clustering the feature descriptors using a standard K-means algorithm. We denote the vocabulary as $W=w_{1}, \cdots, w_{v}$. Finally, an image is expressed by the occurrence rate of visual words in the vocabulary.

Each category is represented by counting the frequency of visual word, $w_{n}$, of all the images in the category. That is, the empirical distribution $p\left(w_{n} \mid\right.$ class $)$ is obtained by summing over the indexes of the training images for the category $I^{\text {class }}$ as follows:

$$
p\left(w_{n} \mid \text { class }\right) \propto \sum_{I^{\text {class }}} \sum_{j=1}^{M^{i}} \delta\left(w_{i j}, w_{n}\right)
$$

With

$$
\delta(x, y)=\left\{\begin{array}{lll}
0, & \text { for } & x \neq y \\
1, & \text { for } & x=y
\end{array}\right.
$$

where $w_{i j}$ describes a quantized visual word of the $j$ th local feature of the $i$ th image. $w_{n}$ denotes a visual word in the vocabulary.

The class-specific distribution $p\left(\operatorname{class} \mid w_{i j}\right)$ can be computed by Bayes theorem,

$$
p(\text { class } \mid w) \propto p(w \mid \text { class }) p(\text { class })
$$

where the prior probability of every class $p$ (class) is computed by training data. As shown in Fig. 5, each category provides its own color histogram according to $p$ (class $\mid w)$.

We define color attention of local features to be equal to the probability of a class given their color values as follows:

$$
\alpha_{t}\left(x_{i j}, \text { class }\right)=p\left(\text { class } \mid w_{i j}\right)
$$

The local color features at the locations $x_{i j}$ are vector quantized into a visual vocabulary, where $w_{i j}$ describes the visual word. If we compute $p($ class $\mid w)$ for all local features in an image we can construct a top-down class-specific color attention map, as shown in Fig. 6. 

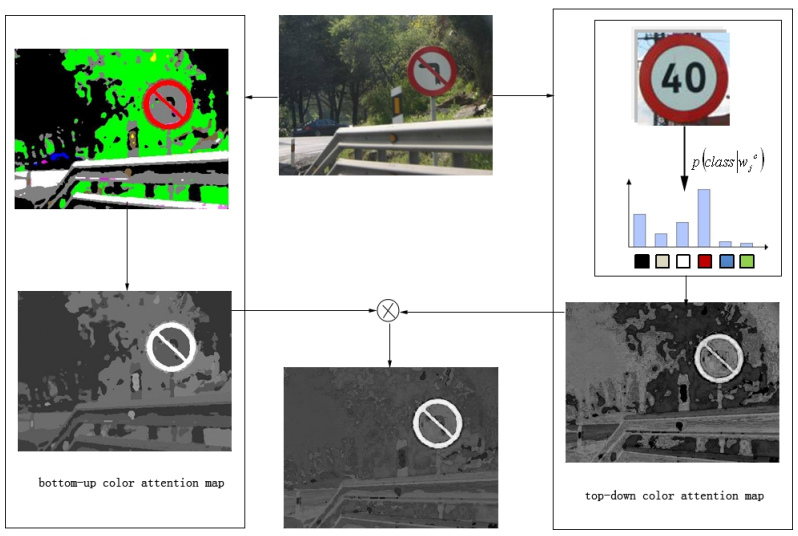

Fig. 6 Detection of a traffic sign in a real world image.

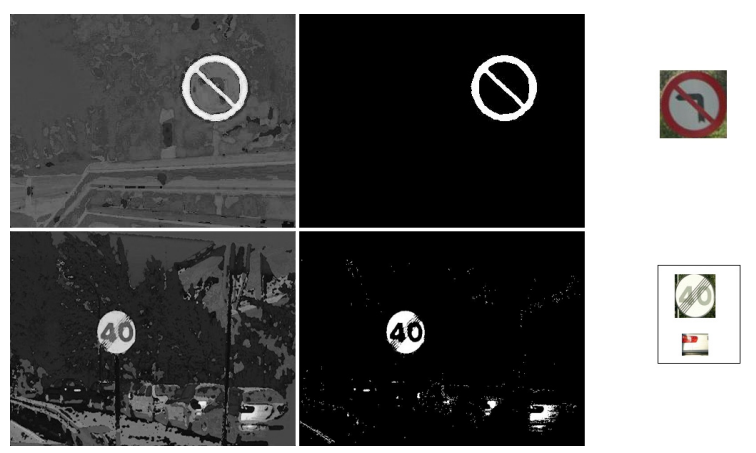

Fig. 7 Saliency segmentation. From left to right are the final saliency maps, the results of saliency segmentation and the candidate road signs in the original images.

\subsection{Saliency Fusion and Segmentation}

The detection of traffic sign is shown in Fig. 6. Both bottomup and top-down saliency maps are normalized and then combined together to construct the final saliency map.

$$
\alpha\left(x_{i j}, \text { class }\right)=\alpha_{b}\left(x_{i j}\right)^{(1-\beta)} \alpha_{t}\left(x_{i j}, \text { class }\right)^{\beta}
$$

where $\alpha_{b}$ is the bottom-up saliency map generated in Sect. 3.1. The parameter $\beta$ is introduced to vary the contribution of top-down and bottom-up saliency maps, where $\beta=0$ indicates only bottom-up saliency and $\beta=1$ means only top-down attention. $\beta$ is learned through cross-validation over the validation set. In our study, we find that $\beta=0.45$ works well for prohibition, warning and obligation signs while $\beta=0.6$ results in best performance for achromatic signs.

The sample adaptive-thresholding method is employed for sign segmentation. The saliency map is first oversegmented by mean-shift and the average saliency is calculated for every region of the whole image. Regions with saliency more than twice of the image average saliency will be set as candidate road signs. Then, the bounding boxes including the candidate road signs are obtained in the original images, as shown in Fig. 7.

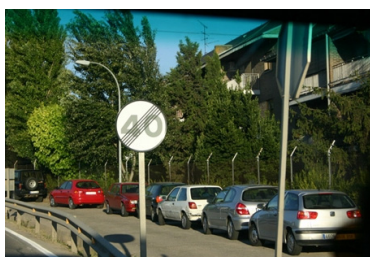

(a)

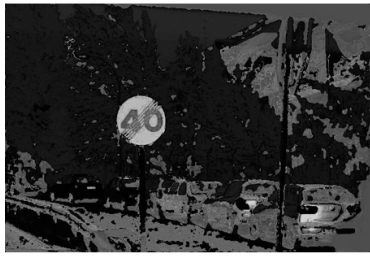

(c)

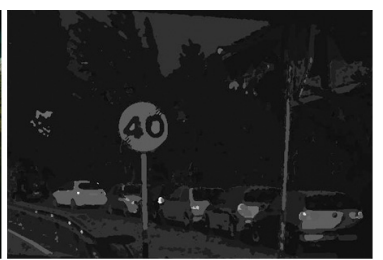

(b)

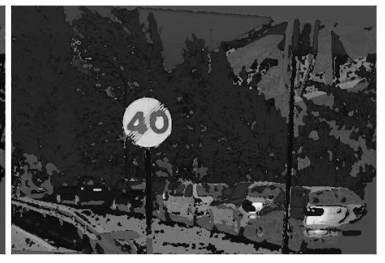

(d)
Fig. 8 Detection of white signs. (a) Original image, (b) bottom-up saliency map, (c) top-down saliency map and (d) the final saliency map.

\subsection{Detection of Achromatic Signs}

Achromatic signs are difficult to detect by the traditional color spaces, such as RGB and HIS; therefore, several classic works about road sign recognition exclude these signs. Note that achromatic colors, such as white, gray and black, are included in the eleven basic colors of the color name descriptor. The color values that they correspond to are captured during the training of color names. During bottom-up saliency map generation, the contrast of achromatic regions is computed the same way as with other color regions. That is, the color name descriptor provides an added advantage of allowing the description of achromatic colors, such as black, gray and white. Sometimes the achromatic regions are not as salient as red or yellow regions because white, gray and black colors can appear in the background. However, the achromatic regions will yield a high saliency value when the background does not contain the same achromatic colors, as shown in Fig. 8. The top-down method detects traffic signs based on color distribution of every category. Therefore, the achromatic signs can be highlighted in a topdown class-specific saliency map when the achromatic category distribution is considered. Then, the bottom-up map and top-down map are combined and followed by normalization. When a region is salient in both bottom-up and topdown maps, it will be more salient in the final saliency map. If it is salient in only one map, it may be more or less salient depending on the saliency value of other regions in the image. The precision of achromatic signs detection is lower than others because of their worse bottom-up saliency maps. However, our method can detect white signs without achromatic decomposition like the work in [2]. One example of white sign detection is shown in Fig. 8 and the quantitative evaluation results of achromatic signs are shown in Fig. 11.

Because of the introduction of top-down color attention, road signs can be classified into prohibition, warning, obligation or achromatic signs during the detection stage. The candidate blobs will only be compared with the signs 


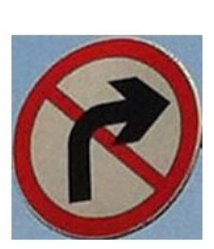

(a)

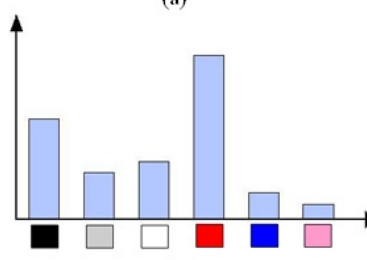

(c)

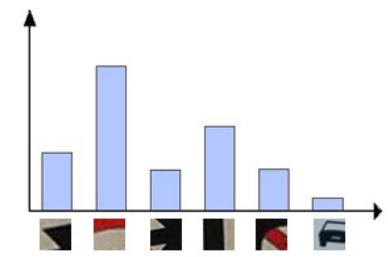

(b)

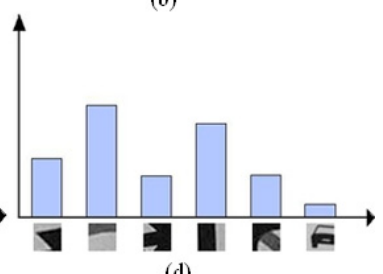

Fig. 9 (a) The sample sign, (b) the histogram generated by a color-shape vocabulary, (c) the histogram generated by a color vocabulary and (d) the histogram generated by a shape vocabulary.

among the same category during the recognition stage.

\section{The Proposed Recognition Method}

Once the candidate blobs are classified into a special category, the recognition process is initiated. The BoW model, which fuses color and shape features together, is employed for image representation. The concept of BoW has been introduced in Sect. 3.2. However, there are two methods to fuse color and shape cues in the bag-of-words framework, early fusion and later fusion. For later fusion, shape and color vocabularies are constructed separately, i.e., we compute $p\left(w^{c} \mid I\right)$ and $p\left(w^{s} \mid I\right)$ and then concatenate the distribution. For early fusion, the cues are fused before vocabulary construction and distribution $p\left(w^{s c} \mid I\right)$ are computed. Early and later fusions result in different image representations and are appropriate for different object categories. Generally, early fusion achieves better performance for colorshape dependence objects while later fusion works well for color-shape independence objects. Road signs are designed with particular colors and shapes. For example, prohibition signs consist of a red circle rim with a white background and black diagram; warning signs have a black triangle rim with a yellow background and black diagram. Because road sign categories are color-shape dependent, early fusion may be superior to later fusion. Figure 9 shows the histogram representations of the sample sign with color, shape and colorshape vocabularies.

$\mathrm{CN}$ and Scale Invariant Feature Transform (SIFT) descriptors are used to express color and shape cues of local features, respectively [28]. The SIFT features are based on the appearance of the object at particular interest points and are invariant to image scale and rotation. They are also robust to changes in illumination, noise and minor changes in viewpoint. As a consequence, each road sign is finally represented by the combination of the two cues, color and shape, as follows:

$$
h\left(w_{n}^{s c} \mid I^{i}\right) \propto \sum_{j=1}^{M^{i}} \delta\left(w_{i j}^{s c}, w_{n}^{s c}\right)
$$

where $w_{n}^{s c}$ is the visual word in the color-shape vocabulary and $w_{i j}^{s c}$ describes a quantized visual word of the $j$ th local feature of the $i$ th image (road sign blob). $h\left(w_{n}^{s c} \mid I^{i}\right)$ is the histogram representation of image $i$, as shown in Fig. 9 .

Every training image is represented by a histogram, which is generated by counting the frequency of visual words in the vocabulary. The histograms are then used to train the classifier. Recognition is implemented by SVMs with Gaussian kernels. For the training process of SVMs, we use the library LIBSVMS. Different one-versusall SVMs classifiers with a Gaussian kernel are used, so that the system can recognize every sign.

\section{Experiments and Results}

\subsection{Dataset}

Until recently, there was no standardized training and test data set for road sign recognition. The main reason is that signs wildly vary between countries and many papers limit their scope to specific sign types. The image quality varies from high-resolution still images to low-resolution frames captured by in-car video cameras. However, a few publicly available road sign data sets exist. In this paper, we employ the data set provided by Spanish researchers MaldonadoBascn $^{\dagger}$. The data set consists of images collected from urban, countryside and highway roads under different weather conditions, covering all types of road signs in Spain. To evaluate how the proposed framework performs for road sign inventory in China, we modify the data set. First, considering the difference of the road signs between China and Spain, we replace the danger signs by the warning signs of China. Second, we divide the data set into four categories: prohibition signs, warning signs, obligation signs and achromatic signs by their function instead of single shape or color. In addition, to evaluate the performance of the detection stage, we provide the ground truth images which are processed by a user.

Both detection and recognition stages need training images. During top-down saliency map generation, the empirical distribution of every category is obtained by training images. The training set consists of 390 road sign blobs (bounding boxes) extracted from 300 images, where 110 samples for prohibition, warning and obligation signs, respectively, and 60 samples for achromatic signs. When training the SVM classifier in the recognition stage, the other 315 negative samples extracted from the same $300 \mathrm{im}$ ages at random are needed. Our test data set includes 500 images with dimensions of $720 * 576$. The number of road signs is 665 .

\footnotetext{
†'http://agamenon.tsc.uah.es/
} 


\subsection{Experimental Setup}

For an input image, we use the mapping learned from Google images in [25] to assign pixels into special color names to form different color clusters. Then, regions are obtained by segmenting a color cluster in a binary image. To reduce the number of regions, we employ a $3 \times 3$ median filter after pixel assignment (the region number can be reduced from $10^{2}$ to $10^{1}$ ).

In our experiment, prohibition, warning, obligation and achromatic signs are included. To obtain better results, we consider the bounding box information and the computing of class-special distribution only from the features inside the bounding boxes. For local feature detection and representation, we employ the multi-scale grid sampling method to obtain the local features, which are expressed by color name descriptors. Then, a universal visual vocabulary representing all road sign categories is computed by clustering the feature descriptors using a standard K-means algorithm. As a result, a visual vocabulary of 180 words is constructed for four types of road signs. Finally, an image is expressed by the visual words in the vocabulary. Every category is represented by counting the frequency of visual words in the images in a special class and provides its own color histogram, as shown in Fig. 5. By computing probability of a special class for each local feature in an image, we can construct a top-down class-special color attention map.

Bottom-up and top-down saliency maps are normalized and then combined to construct the final saliency map. Saliency segmentation is employed to obtain the candidate road signs. In order to reduce the false alarm and save running time in the recognition stage, we have fixed a criterion for discarding inappropriate blobs by their size and aspect ratio. The blobs whose dimensions are delimited between one 20th and two-thirds with respect to the minor dimension of the images or the aspect ratio greater than 1/1.9 and less than 1.9 will be processed.

The recognition stage input, in our case, is a block of $40 \times 40$ pixels in color image for every candidate blob; therefore, the interior of the bounding box is normalized to these dimensions. In the detection stage, the signs are classified into different categories, such as prohibition, warning, obligation or achromatic; thus recognition is processed within each category. The color-shape visual vocabulary is constructed for every category. 60-word vocabularies are constructed for prohibition, warning and obligation signs, while a 30-word vocabulary is designed for achromatic signs.

In the traffic sign data set, there are several triangular signs whose pictograms present a high level of similarity at lower resolution (see the second row of Fig. 10). As the recognition module of our system works with blobs that are normalized to $40 \times 40$ pixels, it is not easy to discern between them. This is the reason, in this paper, as an approximation, we have reorganized these signs within a unique training set, which will be represented by the sign that is shown in the first row of Fig. 10.

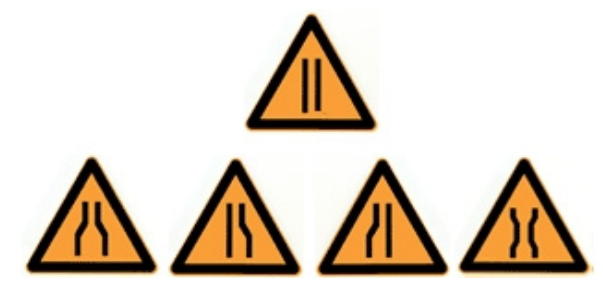

Fig. 10 Set of similar signs. The first row is the representative sign of the set, the second row contains the similar signs.
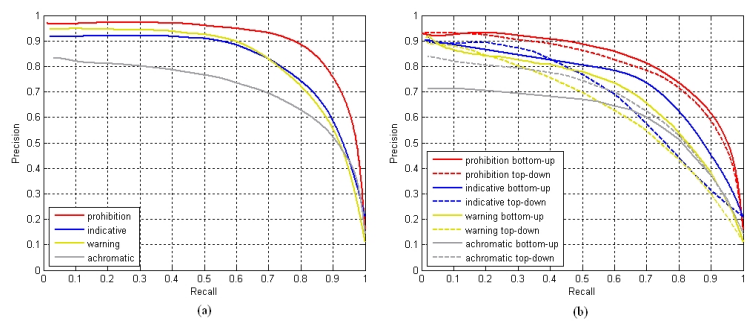

Fig. 11 (a) Precision-recall curves of our method in different types of signs, (b) precision-recall curves of the components in the traffic signs.

\subsection{Experiment Results}

First, we evaluate the performance of the road sign detection algorithm. Both the saliency maps and the final detection results are considered. For saliency map evaluation, a fixed threshold is adopted to obtain a binary segmentation. The segmented image is then compared with the ground truth mask to obtain the precision and recall. The precision is defined as the ratio between the detected true salient pixels and all the detected salient pixels, i.e., truepositive/(truepositive + falsepositive). The recall is defined as the ratio between the detected true salient pixels and salient pixels in the ground truth images, truepositive/positive. To reliably compare how well our saliency detection methods highlight road signs in images, a threshold, $T$, is set from 0 to 255 . As $T$ is changed, different precision-recall pairs are obtained, and a precisionrecall curve is the result. Figure 11 shows the precisionrecall curves of the final saliency maps and the component saliency maps in the traffic signs.

As Fig. 11 (a) shows, our method results in the best performance in prohibition signs. We account this to their more salient red rims. The performance dropped for warning signs and obligation signs. The reason is that both warning and obligation signs include colors (blue or black) that occur frequently in the background, which results in lower saliency value. It is also notable that our method yields promising results in achromatic signs. However, because white and black colors are often present in the background regions, the bottom-up saliency plays less of a role in the detection of achromatic signs. For this reason, the performance of achromatic signs is inferior to other three types of signs.

Figure 11 (b) shows the precision-recall curves of dif- 
Table 1 The precision and recall of different types of signs.

\begin{tabular}{|l|c|c|c|c|}
\hline & Prohibition & Warning & Obligation & Achromatic \\
\hline Precision & 0.9511 & 0.9442 & 0.9318 & 0.8834 \\
\hline Recall & 0.9678 & 0.9348 & 0.9385 & 0.8692 \\
\hline
\end{tabular}

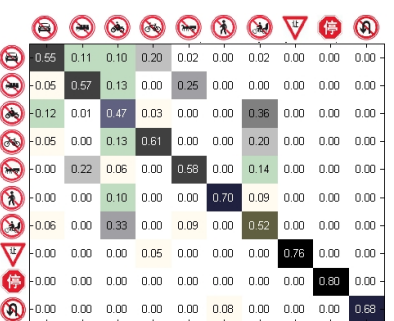

(a)

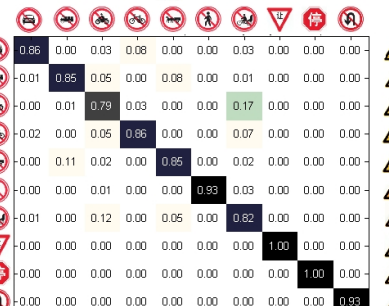

(c)
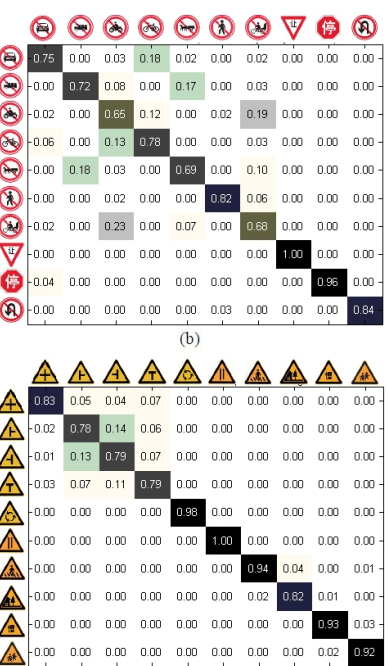

(d)
Fig. 12 Confusion matrices of (a) prohibition signs using CN, (b) prohibition signs using SIFT, (c) prohibition signs using CN and SIFT and (d) warning signs using $\mathrm{CN}$ and SIFT.

ferent components, bottom-up and top-down. We can see that bottom-up saliency maps result in better curves than top-down maps, in particular, when the recall increases. The bottom-up method computes the region saliency by measuring the region's contrast with other regions in the image, which is driven by low-level features and highlights all the salient regions. On the other hand, the top-down method is task-driven and only highlights the potential road sign regions. For this reason, the top-down curves result in better precision when the recall is small ( $T$ is large), and getting worse when the recall is large ( $T$ is small). However, the achromatic sign is one exception where the top-down curve consistently outperforms the bottom-up curve.

To extract candidate road signs, the final saliency map is segmented by the sample adaptive-thresholding method into the binary image. Now we compare the binary images with the ground truth masks. The precision and recall of different types of signs are computed as mentioned above and shown in Table 1.

The presented road sign recognition technique is tested on a diverse set of 500 images that contain 665 different road signs. The accuracy of the proposed algorithm is evaluated from the observation of the recognition results of the road signs that are present in the original images. As for the recognition performances, we investigate the recognition rate of the following three methods: the proposed method that combines the CN and SIFT descriptors and the methods using CN and SIFT separately. Figure 12 shows confusion matrices of some prohibition signs, using three different image representations. The results lead us to several con-
Table 2 Summary of recognition results.

\begin{tabular}{|l|l|}
\hline a) Total number of images & 500 \\
\hline b) Total number of road signs & 665 \\
\hline c) Number of different road signs & 40 \\
\hline d) Recognition of road signs & 548 \\
\hline e) False recognition & 75 \\
\hline f) Number of missing & 27 \\
\hline g) False alarms & 15 \\
\hline
\end{tabular}

clusions. First, the method based on SIFT outperforms the one based on $\mathrm{CN}$ because most of the prohibition signs have much the same color distribution and the discrimination of the CNs is weaker. On the other hand, the shapes of the inner pictogram of signs are more diverse and play a key role in the recognition. Second, there are some relations between the shape and color confusion matrices. If a sign is misclassified as other signs in the color confusion matrix, it tends to be recognized as the same signs in the shape matrix. The results show that road signs are color-shape dependant objects. Third, the method fuses SIFT and CN obtains the best performance because the combination of two types of descriptors provides strong discrimination. Confusion matrix of some warning signs is also given in Fig. 12. The representive sign in Fig. 10 obtains the highest precision because some details of the similar signs are discarded. Note that the sum of every row should be 1; however, there are some exceptions because the signs are recognized as other signs that are not listed in the matrix.

Next, we consider the final results of the proposed method. Correct classification is denoted as "recognition". If the algorithm fails to detect a sign that is present in the test image, then it is denoted as "miss". If it can detect the sign but recognizes it as a wrong sign, then it is designated as "false recognition". Finally, if the system detects a non road-sign object and recognizes it as a road sign, then it is considered a "false alarm". Table 2 summarizes recognition results generated by processing 665 road signs.

The whole road sign recognition algorithm on average takes approximately $1.2 \mathrm{~s}$ for an image with dimensions of $720 * 576$ pixels. The method is implemented in Matlab without any optimization. The simulation platform is a 2.59 GHZ Pentium machine. The whole system is expected to have real-time performance if implemented in the $\mathrm{C}++$ programming language.

\section{Conclusions}

This paper considered the difficulties posed by object recognition in outdoor environments and then proposed a novel framework to detect and recognize traffic signs from real word images. Thus, our system can be useful for the inventory and maintenance of traffic signs.

A regional contrast saliency detection method is proposed to generate bottom-up saliency maps, in which regions with vivid color or with high contrast against the background are enhanced. Furthermore, every type of traffic sign has a unique color distribution, which inspired us to explore 
top-down color attention method to improve detection precision and to classify traffic signs into different categories. For top-down color attention, the bag-of-words (BoW) model is employed to compute special-class distribution, followed by top-down attention map generation. For road sign recognition, color and shape cues are fused in a BoW model before vocabulary generation. A color name descriptor, which can process achromatic signs without decomposition and is invariant to illumination changes to some degree, is used to represent a color cue. On the other hand, a shape cue is expressed by a SIFT descriptor which is invariant to image scale and rotation, and robust to changes in illumination, noise, and minor changes in viewpoint. Each road sign in the training set used here corresponds to a special histogram, which can be used to train a classifier by SVM.

Experimental results indicate that our system is practical because it allows us to recognize different types of traffic signs, i.e., warning, prohibition and obligation signs, and works correctly in difficult situations. Additionally, our method can process white signs without achromatic decomposition. The framework is fast enough for real time applications because all the training steps are processed offline.

\section{References}

[1] C. Seifert, L. Paletta, A. Jeitler, E. Hödl, J.P. Andreu, P. Luley, and A. Almer, "Visual object detection for mobile road sign inventory," Mobile Human-Computer Interaction-MobileHCI 2004, pp.491-495, 2004.

[2] S. Maldonado-Bascn, S. Lafuente-Arroyo, P. Gil-Jimnez, H. GmezMoreno, and F. Lpez-Ferreras, "Road-sign detection and recognition based on support vector machines," IEEE Trans. Intell. Transp. Syst., vol.8, no.2, pp.264-278, June 2007.

[3] M. Kumar, J. Ghorpade, and V.Y. Kulkarni, "Techniques for traffic sign classification using machine learning-A survey," National Conference on Innovative Paradigms in Engineering and Technology (NCIPET-2012), pp.27-32, 2012.

[4] A. Ruta, Y. Li, and X. Liu, "Real-time traffic sign recognition from video by class-specific discriminative features," Pattern Recognit., vol.43, no.1, pp.416-430, Jan. 2010.

[5] J.F. Khan, S.M.A. Bhuiyan, and R.R. Adhami, "Image segmentation and shape analysis for road-sign detection," IEEE Trans. Intell. Transp. Syst., vol.12, no.1, pp.83-96, March 2011.

[6] N. Barnes, G. Loy, and D. Shaw, "The regular polygon detector," Pattern Recognit., vol.43, no.3, pp.592-602, March 2010.

[7] A.C. Le Ngo, L.M. Ang, K.P. Seng, and G. Qiu, "Colour-based bottom-up saliency for traffic sign detection," 2010 International Conference on Computer Applications and Industrial Electronics (ICCAIE), pp.427-431, Dec. 2010.

[8] P. Wang, J. Wang, G. Zeng, and J. Feng, "Salient object detection for searched web images via global saliency," 2012 IEEE Conference on Computer Vision and Pattern Recognition (CVPR), pp.3194-3201, June 2012.

[9] F. Shahbaz Khan, R.M. Anwer, J.van de Weijer, and A.D. Bagdanov, "Color attributes for object detection," 2012 IEEE Conference on Comput. Vis. Pattern Recognit. (CVPR), pp.3306-3313, June 2012.

[10] A. Lorsakul and J. Suthakorn, "Traffic sign recognition using neural network on OpenCV: Toward intelligent vehicle/driver assistance system," 4th International Conference on Ubiquitous Robots and Ambient Intelligence, pp.22-24, 2007.

[11] G. Loy and N. Barnes, "Fast shape-based road sign detection for a driver assistance system," Proc. 2004 IEEE/RSJ International Conference on Intelligent Robots and Systems, 2004.(IROS 2004), vol.1, pp.70-75, Otc. 2004.

[12] D. Xu, Z. Tang, and X. Yan, "Real time road sign detection based on rotational center voting and shape analysis," 2012 International Conference on Mechatronics and Automation (ICMA), pp.1972-1977, 2012.

[13] A. Møgelmose, M. Trivedi, and T. Moeslund, "Vision based traffic sign detection and analysis for intelligent driver assistance systems: Perspectives and survey," IEEE Trans. Intell. Transp. Syst., vol.13, no.4, pp.1484-1497, Dec. 2012.

[14] W. Woong-Jae, L. Minho, and S. Joon-Woo, "Implementation of road traffic signs detection based on saliency map model," IEEE Intelligent Vehicles Symposium, pp.542-547, June 2008.

[15] L. Itti, C. Koch, and E. Niebur, "A model of saliency-basedvisual attention for rapid scene analysis," IEEE Trans. Pattern Anal. Mach. Intell., vol.20, no.11, pp.1254-1259, Nov. 1998.

[16] T. Zhang, J. Lv, and J. Yang, "Road sign detection based on visual saliency and shape analysis," ICIP, pp.3667-3670, 2013.

[17] D. Deguchi, M. Shirasuna, K. Doman, I. Ide, and H. Murase, "Intelligent traffic sign detector: Adaptive learning based on online gathering of training samples," Intelligent Vehicles Symposium (IV), pp.72-77, June 2011.

[18] M. Meuter, C. Nunn, S.M. Gormer, S. Muller-Schneiders, and A. Kummert, "A decision fusion and reasoning module for a traffic sign recognition system," IEEE Trans. Intell. Transp. Syst., vol.12, no.4, pp.1126-1134, Dec. 2011.

[19] Y. Xie, L.F. Liu, C.H. Li, and Y.Y. Qu, "Unifying visual saliency with HOG feature learning for traffic sign detection," Proc. IEEE Intell. Veh. Symp., pp.24-29, June 2009.

[20] G. Overett, and L. Petersson, "Large scale sign detection using HOG feature variants," Proc. IEEE IV Symp., pp.326-331, June 2011.

[21] P. Gil Jiménez, S. Bascón, H. Moreno, S. Arroyo, and F. Ferreras, "Traffic sign shape classification and localization based on the normalized FFT of the signature of blobs and 2D homographies," Signal Process., vol.88, no.12, pp.2943-2955, Dec. 2008.

[22] S. Xu, "Robust traffic sign shape recognition using geometric matching," IET Intell. Transp. Syst., vol.3, no.1, pp.10-18, March 2009.

[23] X. Shen and Y. Wu, "A unified approach to salient object detection via low rank matrix recovery," 2012 IEEE Conference on Computer Vision and Pattern Recognition (CVPR), pp.853-860, 2012.

[24] B. Berlin, and P. Kay, "Basic Color Terms: Their Universality and Evolution," University of California Press, Berkeley, CA, 1969.

[25] J.van de Weijer, C. Schmid, J.J. Verbeek, and D. Larlus, "Learning color names for real-world applications," TIP, vol.18, no.7, pp.1512$1524,2009$.

[26] F.S. Khan, J.van de Weijer, and M. Vanrell, "Modulating shape features by color attention for object recognition," IJCV, 2011.

[27] L. Fei-Fei and P. Perona, "A bayesian hierarchical model for learning natural scene categories," 2005 IEEE Computer Society Conference on Computer Vision and Pattern Recognition (CVPR), pp.524-531, 2005.

[28] D.G. Lowe, "Distinctive image features from scale-invariant keypoints," Int. J. Comput. Vis., vol.60, no.2, pp.91-110, 2004. 

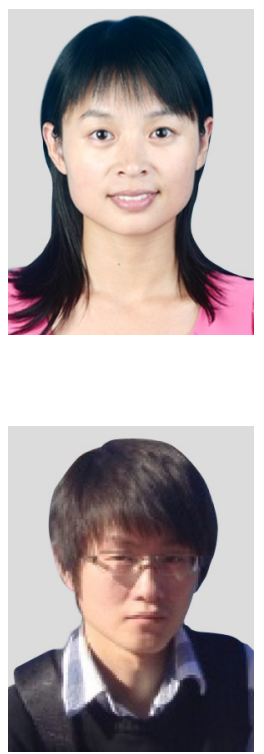

Wei Xu received his bachelor's degree in computer science and technology from Nanjing University of Science and Technology in Nanjing, China, in 2009. He is currently pursuing his Ph.D. degree in the same university. His research interests include salient object detection, color transfer between images, and pavement distress recognition in the intelligent transportation systems.

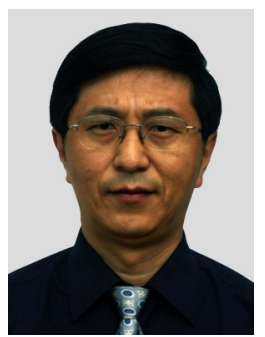

Zhenmin Tang received his M.S. and Ph.D. degree in computer application technology and pattern recognition from Nanjing University of Science and Technology in 1988 and 2002 respectively. He works as a professor in the same university from 1999. His researches include image processing, pattern recognition and intelligent robot.

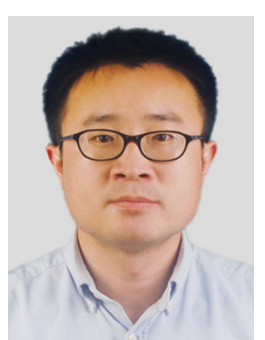

Fan Liu received his B.S. degree in network engineering from Nanjing University of Science and Technology (NUST) in June 2009. $\mathrm{He}$ is currently working towards the Ph.D. degree in computer application technology at Nanjing University of Science and Technology. His research interests include computer vision, image processing, multimedia and pattern recognition. 